Silva, ADV, Cunha, EA \& Araújo, RV (2020). The benefits of integrative and complementary practices in childbirth work. Research, Society and Development, 9(7): 1-16, e614974468.

\title{
Os benefícios das práticas integrativas e complementares no trabalho de parto
}

The benefits of integrative and complementary practices in childbirth work

Los beneficios de lasprácticas integrativas y complementarias eneltrabajo de nacimiento infantil

Recebido:12/05/2020 | Revisado: 18/05/2020 | Aceito: 20/05/2020 | Publicado: 27/05/2020

Adriele Dantas do Val Silva

ORCID: https://orcid.org/0000-0001-9727-7783

Centro Universitário Santo Agostinho, Brasil

E-mail:adrieledovale@gmail.com

Eryca Alencar da Cunha

ORCID: https://orcid.org/0000-0003-3745-050X

Centro Universitário Santo Agostinho, Brasil

E-mail:erycaalencar@hotmail.com

Raquel Vilanova Araújo

ORCID: https://orcid.org/0000-0001-5902-9869

Universidade Federal do Piauí, Brasil

E-mail:raquelvila@outlook.com

\section{Resumo}

Analisar os benefícios das Práticas Integrativas e Complementares no trabalho de parto. Trata-se de uma revisão integrativa. A coleta de dados foi realizada nos meses de fevereiro e março de 2020 nas bases de dados da lilacs, Bdenf, Scielo e Medline. Foram incluídos estudos primários que respondam a pergunta da pesquisa, nos idiomas inglês, português e espanhol e publicados entre os anos de 2006 e 2019, sendo justificado por 2006 ser o ano de implantação da Política de PICs no Brasil. Foram excluídos estudos de revisão, estudos indisponíveis, duplicados, documentos tipo tese, monografia e manuais, obteve-se a amostra inicial de 224 estudos, após leitura dos títulos e resumos obteve 10 estudos que compuseram a amostra final. Foi possível observar que as Práticas Integrativas e Complementares -PICs tem sido mais utilizada na fase ativa do trabalho de parto. Verificou-se que cada uma tem benefícios específicos, no entanto de modo geral, os mais observados foram a redução 
da dor, ansiedade, redução do tempo de trabalho de parto, melhora do conforto, redução de trauma perineal, regulação das contrações uterinas e redução do estresse, calma, relaxamento, concentração e coragem e controle da dor para as mulheres. Destaca-se a importância dos enfermeiros buscarem qualificação na área a fim de prestar uma assistência diferenciada e de qualidade não apenas na obstetrícia mas em nas outras áreas de atuação da enfermagem. Torna-se fundamental o desenvolvimento de pesquisas na área, a fim de comprovar por meio de ensaios clínicos os efeitos das PICs nas possíveis situações.

Palavras-chave: Terapias complementares; Mulher; Parto.

\section{Abstract}

To analyze the benefits of Integrative and Complementary Practices in labor.This is an integrative review. Data collection was carried out in the months of February and March 2020 in the lilacs, Bdenf, Scielo and Medline databases. Primary studies that answer the research question, in English, Portuguese and Spanish, and published between 2006 and 2019, were included, and 2006 is justified as the year of implementation of the PIC Policy in Brazil. Review studies, unavailable studies, duplicates, thesis-type documents, monographs and manuals were excluded. The initial sample of 224 studies was obtained. After reading the titles and abstracts, 10 studies were included in the final sample. It was possible to observe that Integrative and Complementary Practices - PICs were most used in the active phase of labor., reduced labor time, improved comfort, reduced perineal trauma, pressure from uterine contractions and reduced stress, calm, relaxation, concentration and courage and pain control for women. Emphasize the importance of nurses seeking qualification in the area in order to provide differentiated and quality care, not only in obstetrics, but in other areas of nursing practice. It made the development of research in the area fundamental, an end of testing through clinical trials and the effects of possible PICs.

Keywords: Complementarytherapies; Woman; Childbirth.

\section{Resumen}

Analizar los beneficios de las prácticas integradoras y complementarias en el trabajo. Métodos: Esta es una revisión integradora. La recopilación de datos se realizó en los meses de febrero y marzo de 2020 en las bases de datos lila, Bdenf, Scielo y Medline. Se incluyeron los estudios primarios que responden la pregunta de investigación en inglés, portugués y español y se publicaron entre los años 2006 y 2019, y 2006 se justifica como el año de implementación de la Política PIC en Brasil. Se excluyeron los estudios de revisión, los 
estudios no disponibles, los duplicados, los documentos tipo tesis, las monografías y los manuales. Se obtuvo la muestra inicial de 224 estudios. Después de leer los títulos y los resúmenes, se incluyeron 10 estudios en la muestra final. Resultados: se pudo observar que las Prácticas Integrativas y Complementarias - PICs se utilizaron más en la fase activa del trabajo de parto. Se encontró que cada una tiene beneficios promocionales, sin embargo, en general, o las más observadas fueron la reducción del dolor, la ansiedad, tiempo de trabajo reducido, comodidad mejorada, trauma perineal reducido, presión de contracciones uterinas y estrés reducido, calma, relajación, concentración y coraje y control del dolor para las mujeres. Conclusión: Resalte la importancia de que las enfermeras busquen la calificación en el área para brindar atención diferenciada y de calidad, no solo en obstetricia, sino en otras áreas de la práctica de enfermería. Hizo que el desarrollo de la investigación en el área fuera fundamental, un fin de las pruebas a través de ensayos clínicos y los efectos de posibles PIC. Palabras clave:Terapias complementarias; Mujer; Niños.

\section{Introdução}

Utilizadas em diversas situações, para Oliveira \& Merces (2017), o parto é um processo natural e fisiológico marcado por mudanças biológicas, psicológicas e socioculturais na vida da mulher, o que exige acolhimento, cuidado e atenção. E por muito tempo foi visto como um evento familiar, acontecendo em domicilio, com a presença da parteira, comadres e mulheres de sua confiança, que a encorajavam e prestavam cuidados durante o trabalho de parto e pós-parto (Hanun, Mattos, Matão, \& Martins, 2017).

Por meio dos avanços científicos tecnológicos, a mulher deixou de ser protagonista neste cenário, que passou a ser ocupado por medidas extremante medicalizadas, intervenções desnecessárias, e parto cirúrgico sem indicação (Possati, Prates, Cremonese, Scarton, Alves \& Ressel, 2017). Muitas destas situações geravam implicações na vida das mulheres como o medo de uma nova gravidez, embora ainda desejassem ter mais filhos, depressão pós parto, problemas com a amamentação e complicações relacionadas aos procedimentos técnicos (Zanardo, Uribe, Nadal \& Habigzang, 2017)

Diante desta situação, várias medidas foram instituídas com a finalidade de Mudar esta realizada de, dentre estas o Programa de Humanização no Pré-natal e Nascimento (PHPN) implantado com a finalidade de garantir melhorias do acesso, cobertura e qualidade e promover um ambiente acolhedor à mulher, familiares e o recém-nascido, com dignidade e com a instituição de medidas e procedimentos benéficos para o acompanhamento do parto e 
nascimento, evitando intervenções desnecessárias e que não são benéficas à mulher e o bebê (Brasil, 2002).

Outra medida foi a Rede cegonha criada com a intenção de assegurar o acesso, acolhimento e a resolutividade, por meio de um modelo de atenção voltado ao pré-natal, parto e nascimento, puerpério e sistema logístico, que inclui o transporte sanitário e a regulação. Dessa forma a rede cegonha possui tais componentes, como o pré-natal, classificação, acolhimento e vulnerabilidade (Assis, Chagas, Goes, Schafauser, Caitano, \& Marquez 2020).

No contexto atual, o Enfermeiro tem sido peça fundamental no cuidado à mulher durante a gestação, parto e puerpério, de modo incentivar a autonomia, o empoderamento e participação da mulher/ parceiro/família em todo este processo, tendo como vista a melhoria da qualidade da assistência prestada, e dos indicadores relacionados à mortalidade materna infantil. De acordo com é necessário um equilíbrio entre as intervenções necessárias e o processo fisiológico de parir (Alves, Paixão, Fraga, Lírio \& Oliveira 2018).

$\mathrm{Na}$ prática assistencial, o enfermeiro tendo como alicerce o conhecimento baseado em evidência, tem utilizado vários recursos terapêuticos para prestar uma assistência humanizada, de qualidade, e segurança às mulheres, sejam no pré-natal, parto e pós-parto (Borges, Madeira, \& Azevedo, 2011). Dentre estas, as Práticas Integrativas e Complementares (PIC) que tem por objetivo ofertar cuidados de saúde, para racionalização das ações de saúde que estimulam alternativas inovadoras contribuindo para o desenvolvimento sustentável de comunidades, motivando a participação social, podendo ser ofertando tanto pelo SUS como em todos os âmbitos da atenção a saúde (Brasil, 2018).

Dessa maneira as (PIC) tem contribuído positivamente, trazendo inúmeros benéficos como redução da dor, ansiedade, ressaltando a importância da mulher se tornar dona desse momento, tendo consciência da competência do seu corpo e autonomia sobre ele, fazendo com que a mulher desenvolva a autoestima, o autocuidado resgatando assim o seu protagonismo durante o parto (Borges et al, 2011)

A fim de responder a questão norteadora do estudo "Quais os benefícios das práticas integrativas e complementares - PIC no trabalho de parto"elaboraram-seos seguintes objetivos: conhecer as PIC recomendadas no trabalho de parto e seus benefícios e analisar os critérios para recomendação das PIC no trabalho de parto.

A realização de estudos relacionado a temática é essencial para construção de pilares que que serviram de subsidio para a prática diária dos profissionais e para a amplificação do conhecimento relacionado ao uso das Práticas Integrativas e complementares durante o trabalho de parto, além disso com a pesquisa será possível compreender a importância da 
qualificação dos profissionais para atuam em seus diversos campos e dos critérios a serem utilizados para a recomendação das mesmas no trabalho de parto tendo em vista que na atualidade existem várias PIC e que podem ser

\section{Metodologia}

Pesquisas são realizadas para se trazer à luz novos conhecimentos à sociedade como preconizam Pereira, Shitsuka, Parreira \&Shitsuka (2018). O presente estudo trata-se de uma revisão bibliográfica do método revisão integrativa de literatura com abordagem quantitativa que percorreu as seguintes etapas: elaboração da questão de pesquisa; busca na literatura dos estudos primários; coleta de dados; avaliação dos estudos primários; análise e síntese dos resultados e apresentação da revisão (Mendes, Silveira \& Galvão, 2008).

Para responder a pergunta da pesquisa "Quais os benefícios das práticas integrativas e complementares no trabalho de parto?", Foi utilizado a estratégia PICo, o P corresponde a população (mulheres), I, o interesse (benefícios das Práticas integrativas e complementares PIC) e Co (Trabalho de parto).

A coleta de dados foi realizada nos meses de fevereiro e março de 2020 nas bases de dados da Literatura Latino-americana e do Caribe em Ciências da Saúde (LILACS), Base de Dados em Enfermagem (BDENF), ScientificElectronic Library Online (SciELO) e na base de dados da Medical LiteratureAnalysisandRetrieval System Online) (Medline) com os descritores terapias complementares, partoe ComplementaryTherapies, Childbirth, conforme descrito no Quadro 1. 
Research, Society and Development, v. 9, n.7, e614974468, 2020

(CC BY 4.0) | ISSN 2525-3409 | DOI: http://dx.doi.org/10.33448/rsd-v9i7.4468

Quadro 1 - Estratégia de busca, bases de dados utilizados e amostragem.

\begin{tabular}{|c|c|c|c|}
\hline Estratégia & Base de dados & $\begin{array}{l}\text { Após leitura } \\
\text { dos títulos }\end{array}$ & Amostra \\
\hline Terapias Complementares AND Parto & Lilacs (25) & 17 & 6 \\
\cline { 2 - 4 } & BDENF (9) & 9 & 1 \\
\hline Terapias Complementares OR Parto & Lilacs (63) & 5 & 2 \\
\cline { 2 - 4 } & Bdenf (10) & 1 & 1 \\
\hline Complementary Mesh Therapies Mesh Childbirth & Medline (105) & 32 & 0 \\
\hline Terapias complementares AND parto & Scielo (12) & 10 & 0 \\
\hline Total & $\mathbf{2 2 4}$ & $\mathbf{7 4}$ & $\mathbf{1 0}$ \\
\hline
\end{tabular}

Fonte: Silva, ADV, Cunha, EA \& Araujo, RV 2020.

No Quadro 1 estão expostos estratégia de busca, base de dados e amostragem. Na estratégia de busca foram utilizadas as palavras chaves, terapias complementares e parto com os operadores booleanos AND e OR para pesquisa nas bases de dados Lilacs, Bdenf e Scielo e Mesh para Complementary Therapies e Childbirth para busca na Medline. Foram incluídos estudos primários que respondam a pergunta da pesquisa, publicados nosidiomas inglês, português e espanhol e publicados entre os anos de 2006 e 2019. O recorte temporal justificase pelo fato de em 2006 ter sido o ano de implantação da Política Nacional de Práticas integrativas e Complementares -PNPIC no Sistema Único de Saúde (SUS) (Brasil, 2006). Foram excluídos estudos de revisão da literatura, textos incompletos, duplicados, documentos tipo tese, monografia, dissertação e manuais.

$\mathrm{Na}$ amostra inicial tem-se um total de 224 artigos, após a leitura dos títulos e resumos, obteve a amostra final de 10 estudos que foram lidos na integra, analisados, interpretados, discutidos e confrontado com luz teórica acerca da temática em discussão. Os dados foram extraídos utilizando um formulário construído pelos pesquisadores que contempla informações referentes ao ano de publicação dos estudos, periódico, idioma, título, objetivo, metodologia, os benefícios das PICs no trabalho de parto e conclusão. 


\section{Resultados}

Quadro 2 - Caracterização dos artigos conforme nome dos autores, título dos estudos e objetivo. Teresina, PI, 2020.

\begin{tabular}{|c|c|c|c|}
\hline $\mathbf{N}$ & Autor/ano & Título & Objetivo \\
\hline 01 & $\begin{array}{l}\text { Cavalcanti HB, } \\
\text { Gabrielloni B, } \\
\text { (2019) }\end{array}$ & $\begin{array}{l}\text { Terapias complementares no } \\
\text { trabalho de parto: ECR }\end{array}$ & $\begin{array}{l}\text { Avaliar o efeito do banho quente de chuveiro e exercício } \\
\text { perineal com bola suíça isolados e de forma combinada, } \\
\text { sobre a percepção da dor, ansiedade e progressão do } \\
\text { trabalho de parto. }\end{array}$ \\
\hline 02 & $\begin{array}{l}\text { Manfetoni RS, } \\
\text { JS(2019) }\end{array}$ & $\begin{array}{l}\text { Efetividade da auriculoterapia } \\
\text { sobre a dor no trabalho de } \\
\text { parto: ECR }\end{array}$ & $\begin{array}{l}\text { Avaliar a efetividade da auriculoterapia sobre a dor na } \\
\text { fase ativa do trabalho de parto. }\end{array}$ \\
\hline 03 & $\begin{array}{l}\text { ManfetoniJ, Jorge } \\
\text { S (2018) }\end{array}$ & $\begin{array}{l}\text { Efeitos da auriculoterapia no } \\
\text { tempo de trabalho de parto e } \\
\text { taxa de cesárea: ECR }\end{array}$ & $\begin{array}{l}\text { Avaliar os efeitos da auriculoterapia sobre o tempo de } \\
\text { trabalho de parto e taxa de cesárea. }\end{array}$ \\
\hline 04 & $\begin{array}{l}\text { Henrique G, } \\
\text { Cavalcante MB } \\
\text { (2016) }\end{array}$ & $\begin{array}{l}\text { Hidroterapia e bola suíça no } \\
\text { trabalho de parto: ECR }\end{array}$ & $\begin{array}{l}\text { Conhecer a influência do banho quente e exercício } \\
\text { perineal com bola suíça, de forma isolada e combinada, } \\
\text { sobre a progressão do trabalho de parto. }\end{array}$ \\
\hline 05 & $\begin{array}{l}\text { Manfetoni \& } \\
\text { Shimo (2016) }\end{array}$ & $\begin{array}{l}\text { Efeitos da acupressao sobre a } \\
\text { dor no trabalho de parto: ECR }\end{array}$ & $\begin{array}{l}\text { Analisar os efeitos da acupressao no ponto sanyinjiao } \\
\text { sobre a dor na fase ativa do TP, em gestantes atendidas } \\
\text { em maternidade pública. }\end{array}$ \\
\hline 06 & $\begin{array}{l}\text { Mafetoni \& } \\
\text { Shimo (2015) }\end{array}$ & $\begin{array}{l}\text { Efeitos da acupressao na } \\
\text { evolução do parto e taxa de } \\
\text { cesárea: ECR }\end{array}$ & $\begin{array}{l}\text { Analisar os efeitos da acupressao no ponto BP6 no tempo } \\
\text { de TP e na taxa de cesárea, em parturientes atendidas em } \\
\text { maternidade pública. }\end{array}$ \\
\hline 07 & $\begin{array}{l}\text { Mafetoni, } \\
\text { Rodrigues, Jacob } \\
\text { \& Shimo(2018) }\end{array}$ & $\begin{array}{l}\text { Efetividade da auriculoterapia } \\
\text { sobre a ansiedade no trabalho } \\
\text { de parto: ECR }\end{array}$ & $\begin{array}{l}\text { Avaliar a efetividade da auriculoterapia sobre a ansiedade } \\
\text { de mulheres durante o TP. }\end{array}$ \\
\hline 08 & $\begin{array}{l}\text { Santana GF, } \\
\text { Quintana } \\
\text { M(2013) }\end{array}$ & $\begin{array}{l}\text { Efeito do banho de chuveiro } \\
\text { no alívio da dor em } \\
\text { parturientes na fase ativa do } \\
\text { trabalho de parto }\end{array}$ & $\begin{array}{l}\text { Avaliar o efeito do banho de chuveiro no alívio da dor, } \\
\text { durante a fase ativa do trabalho de parto. }\end{array}$ \\
\hline 09 & $\begin{array}{l}\text { Silva \& Oliveira } \\
(2006) \text {. }\end{array}$ & $\begin{array}{l}\text { O efeito do banho de imersão } \\
\text { na duração do trabalho de parto }\end{array}$ & $\begin{array}{l}\text { Identificar a influência do banho de imersão na duração do } \\
\text { primeiro período clínico do parto e na frequência e duração } \\
\text { das contrações uterinas. }\end{array}$ \\
\hline 10 & $\begin{array}{l}\text { Lara M, Cesar G\& } \\
\text { Barbieri } \\
(2020)\end{array}$ & $\begin{array}{l}\text { Vivência de mulheres em } \\
\text { trabalho de parto com o uso de } \\
\text { essências florais }\end{array}$ & $\begin{array}{l}\text { Descrever a vivência de mulheres submetidas ao uso de } \\
\text { essências florais como terapia não farmacológica para o } \\
\text { alívio da dor e ansiedade durante o trabalho de parto. }\end{array}$ \\
\hline
\end{tabular}

Fonte: Silva ADV, Cunha, EA \& Araujo RV 2020.

No Quadro 2 tem-se a apresentação dos artigos selecionados para o estudo conforme nome dos autores, ano, títulos e objetivos com a finalidade de facilitar o desenvolvimento da 
discussão. A partir do estudo dos artigos estabeleceram-se variáveis relevantes para observação das produções científicas relacionadas à pesquisa, conforme descrito na Tabela 1 .

Tabela 1 - Distribuição das produções científicas segundo o ano de publicação, periódico, abordagem metodológica e região geográfica. Teresina, 2020.

\begin{tabular}{|c|c|c|}
\hline Ano & $\mathbf{N}$ & $\%$ \\
\hline 2006 & 1 & 9,1 \\
\hline 2013 & 1 & 9,1 \\
\hline 2015 & 1 & 9,1 \\
\hline 2016 & 2 & 18,1 \\
\hline 2018 & 2 & 18,1 \\
\hline 2019 & 2 & 18,1 \\
\hline 2020 & 1 & 9,1 \\
\hline \multicolumn{3}{|l|}{ Periódico } \\
\hline Texto \& Contexto Enfermagem & 1 & 9,1 \\
\hline Revista Mineira de Enfermagem & 1 & 9,1 \\
\hline Acta Paulista de Enfermagem & 1 & 9,1 \\
\hline Revista Latino-Americana de Enfermagem & 2 & 18,1 \\
\hline Revista Saúde Pública & 1 & 9,1 \\
\hline Revista de Pesquisa: Saúde é Fundamental Online & 1 & 9,1 \\
\hline Revista da Escola de Enfermagem da USP & 1 & 9,1 \\
\hline Revista Dor & 1 & 9,1 \\
\hline Revista Gaúcha de Enfermagem & 1 & 9,1 \\
\hline \multicolumn{3}{|l|}{ Tipo de estudo } \\
\hline Ensaio clínico randomizado (ECR) & 8 & 80 \\
\hline Pesquisa qualitativa & 2 & 20 \\
\hline \multicolumn{3}{|l|}{ Local de Realização do Estudo } \\
\hline Sudeste & 10 & 100 \\
\hline Sul & 0 & \\
\hline Centro-Oeste & 0 & \\
\hline
\end{tabular}




$\begin{array}{ll}\text { Norte } & 0 \\ \text { Nordeste } & 0\end{array}$

Fonte: Silva ADV, Cunha EA \& Araujo RV 2020.

Na Tabela 1, observa-se que o maior numero de publicações ocorreu no ano de 2016 $3(27,7 \%)$ e na Revista Latino-Americana de Enfermagem 2(18,8\%). Quanto ao tipo de estudo, verifica-se maior destaque de estudos do tipo ensaio clinico randomizado $8(80 \%)$ e desenvolvidos na região sudeste do Brasil na cidade de São Paulo.

Quadro 3 - Uso das Práticas Integrativas e complementares nos respectivos momentos do trabalho de parto, pródromos, fase latente e fase ativa.

\begin{tabular}{|c|c|c|c|}
\hline \multicolumn{5}{|c|}{ Praticas integrativas e complementares } \\
\hline Pródomos TP & Fase latente TP & Fase ativa TP & $\mathbf{n}^{\mathbf{0}}$ \\
\hline$*$ & $*$ & Auriculoterapia & $02,03 \mathrm{e} 07$ \\
\hline$*$ & $*$ & Acupressao & 05 e 06 \\
\hline$*$ & $*$ & Banho de chuveiro & 08 \\
\hline$*$ & $*$ & Banho de imersão & 09 \\
\hline$*$ & $*$ & Essências florais & 04 \\
\hline$*$ & $*$ & Hidroterapia & 04 \\
\hline$*$ & $*$ & Bola suíça & 04 \\
\hline
\end{tabular}

Siglas: TP(Trabalho de parto); *( Nenhuma prática encontrada nessa fase do TP). Fonte: Silva, ADV, Cunha, EA \& Araujo, RV 2020.

No Quadro 3, tem-se a descrição em relação ao uso das Práticas Integrativas e complementares utilizadas nos respectivos momentos do trabalho de parto, pródromos, fase latente e fase ativa. Foi possível observar o uso das práticas integrativas apenas na fase ativa do trabalho de parto, sendo as mais utilizadas, auriculoterapia, acupressao, dentre outras. 
Quadro 4 - Benefícios das Práticas Integrativas e Complementares utilizadas no trabalho de parto.

\begin{tabular}{|c|c|c|}
\hline \multicolumn{3}{|c|}{ Benefícios das práticas integrativas e complementares (PICs) no trabalho de parto } \\
\hline PICs & Benefícios & Recomendação \\
\hline Auriculoterapia & $\begin{array}{l}\text { - } \text { Redução da dor } \\
\text { - } \text { Redução da ansiedade } \\
\text { - } \\
\begin{array}{l}\text { Redução do tempo de trabalho de } \\
\text { parto }\end{array}\end{array}$ & $\begin{array}{l}\text { IG } \geq 37 \text { semanas } \\
\text { TP espontâneo } \\
\text { Dilatação Cervical } \geq 4 \mathrm{~cm} \\
\text { Duas ou mais contrações em } 10 \text { min }\end{array}$ \\
\hline Hidroterapia & $\begin{array}{ll}\text { - } & \text { Alivio da dor e reduz ansiedade } \\
\text { - } & \text { Aumenta o conforto } \\
\text { - } & \text { Proporciona sentimentos positivos } \\
& \text { durante o TP } \\
\text { - } & \text { Acelera o trabalho de parto } \\
\text { - } & \text { Reduz trauma perineal } \\
\text { - } & \text { Redução do estresse } \\
\text { - } & \text { Regulação das CU* }\end{array}$ & $\begin{array}{l}\text { Idade mínima de } 18 \text { anos } \\
\text { Feto único e vivo } \\
\text { Apresentação cefálica } \\
\text { Ausência de patologia clínica ou obstétrica } \\
\text { Dilatação cervical entre } 3 \text { a } 8 \mathrm{~cm} \\
\text { IG a termo. }\end{array}$ \\
\hline Acupressao & $\begin{array}{ll}\text { - } & \text { Alívio da dor } \\
\text { - } & \text { Menor tempo de TP }\end{array}$ & $\begin{array}{l}\mathrm{IG} \geq 37 \text { semanas } \\
\text { Dilatação cervical } \geq 4 \mathrm{~cm} \\
\text { Duas ou mais contrações em } 10 \mathrm{~min} .\end{array}$ \\
\hline Banho de imersão & $\begin{array}{ll}\text { - } & \text { Alivio da dor } \\
\text { - } & \text { Maior dilatação cervical }\end{array}$ & $\begin{array}{l}\text { Ser nulípara } \\
\text { Gestação a termo } \\
\text { Feto com apresentação cefálica fletida; } \\
\text { Não ter intercorrências clínicas ou obstétricas; } \\
\text { Dilatação cervical } \leq 6 \text { centímetros } \\
\text { Bolsa das águas íntegra ou rota } \\
\text { espontaneamente } \\
\text { Período < à } 6 \text { horas antes da admissão DU com } \\
\text { duas ou mais contrações em } 10 .\end{array}$ \\
\hline Banho de chuveiro & - Alívio da dor & $\begin{array}{l}\text { Primigestas } \\
\text { Alfabetizadas } \\
\text { Feto único em posição cefálica } \\
\text { Gravidez de baixo risco } \\
\text { IG } \geq 37 \text { semanas } \\
\text { Dilatação cervical entre } 4 \text { e } 5 \mathrm{~cm} \\
\text { Dinâmica uterina adequada para fase TP } \\
\text { Trabalho de parto espontâneo }\end{array}$ \\
\hline Essência Floral & $\begin{array}{ll}\text { - } & \text { Calma } \\
\text { - } & \text { Relaxamento } \\
\text { - } & \text { Concentração } \\
\text { - } & \text { Coragem } \\
\text { - } & \text { Controle da dor }\end{array}$ & $\begin{array}{l}\text { Risco obstétrico habitual } \\
\text { Fase ativa do trabalho de } \\
\text { Parto }\end{array}$ \\
\hline Bola suíça & 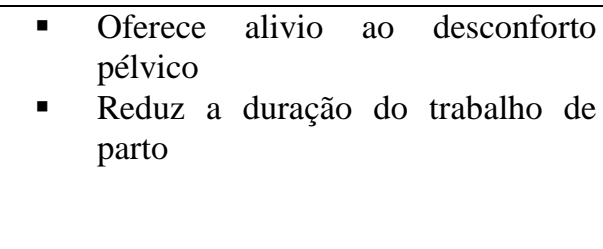 & $\begin{array}{l}\text { Idade mínima de } 18 \text { anos } \\
\text { Feto único e vivo } \\
\text { Apresentação cefálica } \\
\text { Ausência de patologia clínica ou obstétrica } \\
\text { Dilatação cervical entre } 3 \text { a } 8 \mathrm{~cm} \\
\text { IG a termo. }\end{array}$ \\
\hline
\end{tabular}

*CU-Contração uterina.

yr*IG-Idade Gestacional.

Fonte: Silva, ADV, Cunha, EA \& Araujo, RV 2020.

No Quadro 4, tem-se a apresentação em relação ao uso das PICs, seus benéficos e as recomendações. Foi possível observar que as PICs proporcionam inúmeros benefícios, e que o 
alívio da dor se encontra em quase todas, e que quando se trata das recomendações a maioria orienta que seja realizada com $\mathrm{IG} \geq 37$ semanas.

\section{Discussão}

Segundo Mafetoni, Rodrigues, Silva, Jacob \& Shimo(2019) a auriculoterapia possui pontos característicos para o tratamento de distócias obstétricas, redução do período expulsivo e dor do parto tornando-se uma alternativa de cuidado para parturientes através de técnica não invasiva. Em relação a dor o autor relata que a redução nos escores de Escala Analógica e Visual (EVA) não é grande, mas devido a dor na fase ativa ser progressiva e não aumentar, o cuidado dispensado pode ser considerado eficaz. O autor trás a mesma ideia quando em outro estudo se refere a acupressão sobre a dor no trabalho de parto, aplicada no ponto BP6, localizada no meridiano baço-pâncreas, a quatro dedos do paciente acima da ponta do maléolo interno (Manfetoni \& Shimo, 2016).

Outra prática eficiente no alívio da dor no trabalho de parto é o uso de essências florais, partindo do princípio que as emoções positivas promovem saúde e as negativas geram doenças a terapia floral tem a função de restaurar o estado positivo da personalidade do ser humano utilizando essências de flores para cuidar de aspectos mentais, emocionais e comportamentais das pessoas. E durante o trabalho de parto proporcionam benefícios como calma, relaxamento e concentração para as parturientes, favorecendo o controle da dor através dessas emoções, contribuindo para que se tornem protagonistas do seu parto, por sentirem-se mais confiantes no processo de evolução do trabalho de parto, demonstrando-se mais corajosas em relação à dor (Lara, Magaton, Cesar, Gabrielloni \& Barbieri, 2020).

E ainda se tratando do alívio da dor Santana, Gallo, Ferreira, Quintana \& Marcollin (2013) afirma que o banho de chuveiro é um método não farmacológico muito benéfico ao alívio da dor no trabalho de parto, sem efeitos adversos e tem boa aceitação pelas parturientes, e para que tenha o efeito esperado é necessário que a temperatura da água esteja entre 37 e $38^{\circ} \mathrm{C}$, tendo a parturiente que permanecer no banho por no mínimo 20 minutos, isto promovera vasodilatação e redistribuição do fluxo sanguíneo, relaxando assim a musculatura, mas ressalta que é contraindicado no caso de pacientes com hipotensão arterial, devido a vasodilação periférica que a água quente proporciona.

Já, Silva \& Oliveira (2008) e Henrique, Gabrielloni, Cavalcante, Melo \& Barbieri (2016) relatam que o banho de imersão e hidroterapia aplicada na região lombo-sacro também alivia a dor, e trás outros benefícios junto a ele como a redução do uso de fármacos ou a 
postergação dos mesmos, mas salientam que o banho não é indicado para parturientes com dilatação inferior a $5 \mathrm{~cm}$, uma vez que quando realizado precocemente há uma necessidade maior de analgesia, certamente devido ao trabalho de parto mais prolongado.

Henrique et al (2016) e Cavalcante, Henrique, Brasil, Gabrielloni \& Barbieri (2019) faz relação do banho quente com o uso da bola suíça e relata que quando associados os benefícios são ainda maiores, como maior ocorrência de parto normal, maior progressão de dilatação cervical, melhor evolução da descida da apresentação fetal e redução no tempo do trabalho de parto. Estes resultados podem servir de estimulo aos profissionais obstétricos, para que a terapia seja utilizada, pois além dos benefícios não requer altos investimentos.

E se tratando de menor tempo de trabalho de parto a auriculoterapia e acupressao também promovem esse benefício, segundo Manfetoni, Jacob, Jorge \& Shimo (2018)alguns pontos auriculares quando estimulados são capazes de ativar o sistema nervoso autônomo com predomínio do sistema nervoso simpático, encarregado pelas contrações uterinas, distensão do segmento inferior do útero e colo uterino, que pode reduzir o tempo de trabalho de parto. E em relação a acupressao quando aplicada ao ponto BP6 também diminui o tempo de trabalho de parto (Mafetoni \& Shimo, 2015).

O processo do parto desperta muita ansiedade e estresse a mulher durante toda a gestação, principalmente pelo medo do parto, e a auriculoterapia se mostra eficaz na redução da ansiedade na fase ativa do trabalho de parto (Mafetoni, Rodrigues, Jacob \& Shimo, 2018). A hidroterapia também promove a diminuição da ansiedade nesse período, devido ao banho propiciar sensação de bem estar, relaxamento, e maior satisfação decorrente da liberdade (Henrique et al., 2016, Cavalcante et al, 2019).

Em estudo sobre auriculoterapia e acupressao na evolução do parto e taxa de cesárea, os resultados não são claros, mas essas intervenções não pioraram esses indicativos, existem boas evidências relacionadas a essas práticas que podem ser estudas e colocadas em prática por enfermeiros obstetras que sejam capacitados com a finalidade de aumentar as alternativas de cuidados para suas parturientes (Mafetoni \& Shimo, 2015, Manfetoni et al, 2018)

Diante do exposto pode-se observar que as práticas integrativas e complementares no trabalho de parto são totalmente direcionadas para o bem estar materno e consequentemente do bebê, promovendo melhores condições a parturiente, bem como a redução da ansiedade, relaxamento, alívio da dor, regulação do estresse, benefícios estes que fazem com que a mulher tome consciência do seu processo de parturição e seja protagonista do seu parto. 


\section{Considerações Finais}

Diante disso é possível observar que as Práticas Integrativas e Complementares-PICs tem sido mais utilizada na fase ativa do trabalho de parto, tendo como destaque a auriculoterapia, acupressao, banho de chuveiro, banho de imersão, essências florais, hidroterapia e bola suíça. A auriculoterapia teve benefícios na redução da dor, ansiedade, redução do tempo de trabalho de parto; A hidroterapia ajudou no alivio da dor e redução da ansiedade, melhora do conforto, aceleração do trabalho de parto, redução de trauma perineal, regulação das contrações uterinas e redução do estresse; A acupressao contribui para o alívio da dor e redução do tempo de TP; O banho de chuveiro e imersão ajudou no alivio da dor e na dilatação cervical; Bola suíça foi usada com a finalidade de provocar melhorar o desconforto pélvico e reduz a duração do trabalho de parto e os Florais foram utilizados com a finalidade de trazer mais calma, relaxamento, concentração, coragem e controle da dor para as mulheres;

Do mesmo modo verificou-se que os profissionais seguem as recomendações em relação à indicação e contraindicação para o uso de determinada PIC, neste fundamental o conhecimento e experiência em relação ao á aplicabilidade destas terapias na prática assistencial, logo como qualquer outro tipo de terapia, se utilizada de maneira adequada é possível ocasionar danos tanto á mulher bem como ao feto.

Deste modo destaca-se que a cada dia vem sendo ampliado o uso das PICs na rede de assistência à saúde, tanto pública como privada e em diversas cenários como obstetrícia, pediatria, pedagogia, psiquiatria, dentre outras e com inúmeras finalidades dentre elas preventiva, terapêutica ou mesmo como estilo de vida. As PICs também vem sendo incentivado com a finalidade de desenvolver a autonomia dos profissionais e dos adeptos, já repercute na melhora da qualidade de vida e reduz o uso de medicações. Além disso é reconhecida como um método que visa compreender o indivíduo em sua completude, de modo holístico, integral e humanizado.

Por ser uma um campo da saúde que está em crescimento, destaca-se a importância dos enfermeiros buscarem qualificação na área, a fim de prestar uma assistência diferenciada e de qualidade não apenas na obstetrícia mas em várias de atuação da enfermagem. Outro ponto a ser considerado é a necessidade do desenvolvimento de pesquisas na área, já que ainda são tímidas as produções cientificas, em especial estudos clínicos. 


\section{Referências}

Alves, TTM, Paixão, GPN, Fraga, CDS, Lírio, JGS \& Oliveira, FA. (2018). Atuação da enfermeira obstetra no desenrolar do trabalho de parto e parto. Revista de Enfermagem e Atenção à Saúde, 7(1).

Assis, TR, Chagas, VO, Goes, RDM, Schafauser, NS, Caitano, KG \& Marquez, RA. (2019). Implementação da Rede Cegonha em uma Regional de Saúde do estado de Goiás: o que os indicadores de saúde mostram sobre atenção materno-infantil?.

Brasil (2002). Ministério da saúde. Secretaria Executiva Humanização no Pré-natal e nascimento. Brasília.

Brasil (2018) Ministério da Saúde. Secretaria de Atenção à Saúde. Departamento de Atenção Básica. Manual de implantação de serviços de práticas integrativas e complementares no SUS / Ministério da Saúde, Secretaria de Atenção à Saúde, Departamento de Atenção Básica. Brasília.

Borges, MR, Madeira, LM \& Azevedo, VMGDO. (2011). As práticas integrativas e complementares na atenção à saúde da mulher: uma estratégia de humanização da assistência no Hospital Sofia Feldman. Revista Mineira de Enfermagem, 15(1), 105-113.

Cavalcanti, ACV, Henrique, AJ, Brasil, CM, Gabrielloni, MC\& Barbieri, M. (2019). Terapias complementarias em eltrabajo de parto: ensayo clínico randomizado. Revista Gaúcha de Enfermagem, 40.

Hanum, SDP, Mattos, DVD, Matão, MEL \& Martins, CA. (2017). Estratégias não farmacológicas para o alívio da dor no trabalho de parto: efetividade sob a ótica da parturiente. Rev. enferm. UFPE online, 3303-3309.

Henrique, AJ, Gabrielloni, MC, Cavalcanti, ACV, Souza Melo, P\& Barbieri, M. (2016). Hidroterapia e bola suíça no trabalho de parto: ensaio clínico randomizado. Acta Paulista de Enfermagem, 29(6), 686-692. 
Lara, SRG, Silva Magaton, APF, Cesar, MBN, Gabrielloni, MC\& Barbieri, M. (2020).

Vivência de mulheres em trabalho de parto com o uso de essências florais. Revista de Pesquisa: Cuidado é Fundamental, 161-167.

Mafetoni, RR \& Shimo, AKK. (2015). Efeitos da acupressão na evolução do parto e taxa de cesárea: ensaio clínico randomizado. Revista de Saúde Pública, 49, 9.

Mafetoni, RR \& Shimo, AKK. (2016). The effects of acupressure on labor pains during child birth: randomized clinical trial. Revista latino-americana de enfermagem, 24.

Mafetoni, RR, Jacob, LMS, Jorge, HMF\&Shimo, AKK. (2018). Efeitos da auriculoterapia no tempo de trabalho de parto e taxa de cesárea: ensaio clínico randomizado. REME rev. min. enferm, e-e.

Mafetoni, RR, Rodrigues, MH, Jacob, LMDS \& Shimo, AKK. (2018). Effectiveness of auriculo therapy on anxiety during labor: a randomized clinical trial. Revista latino-americana de enfermagem, 26.

Mafetoni, RR, Rodrigues, MH, Silva, FMBD, Jacob, LMDS \& Shimo, AKK. (2019).

Efetividade da auriculoterapia sobre a dor no trabalho de parto: ensaio clínico randomizado. Texto \& Contexto-Enfermagem, 28.

Mendes, KDS, Silveira, RCDCP \& Galvão, CM. (2008). Revisão integrativa: método de pesquisa para a incorporação de evidências na saúde e na enfermagem. Texto \& ContextoEnfermagem, 17(4), 758-764.

Oliveira, MC, Merces, MC. Magno Conceição das Merces. (2017). Percepções sobre violências obstétricas na ótica de puérperas Revenferm UFPE online., Recife, 11(Supl. 6): 2483-9, jun.

Pereira, AS, Shitsuka, DM, Parreira, FJ \& Shitsuka, R. (2018). Metodologia da pesquisa científica. [e-book]. Santa Maria. Ed. UAB/NTE/UFSM. Disponível em: https://repositorio.ufsm.br/bitstream/handle/1/15824/Lic_Computacao_MetodologiaPesquisa-Cientifica.pdf?sequence $=1$. 
Possati, AB, Prates, LA, Cremonese, L, Scarton, J, Alves, CN \&Ressel, LB. (2017).

Humanização do parto: significados e percepções de enfermeiras. Escola Anna Nery, 21(4).

Santana, LS, Gallo, RBS, Ferreira, CHJ, Quintana, SM \&Marcolin, AC. (2013). Efeito do banho de chuveiro no alívio da dor em parturientes na fase ativa do trabalho de parto. Revista Dor, 14(2), 111-113.

Silva, FMBD\& Oliveira, SMJVD. (2006). El efecto del baño de inmersión enladuración del trabajo de parto. Revista da Escola de Enfermagem da USP, 40(1), 57-63

Zanardo, GLDP, Uribe, MC Nadal, AHRD \& Habigzang, LF. (2017). Violencia obstétrica enbrasil: una revisión narrativa. Psicologia \& sociedade, 29.

\section{Porcentagem de contribuição de cada autor no manuscrito}

Adriele Dantas do Val Silva- 10\%

Eryca Alencar da Cunha - 10\%

Raquel Vilanova Araújo - 80\% 